\title{
Variables Influencing the Depth of Conscious Sedation in Plastic Surgery: A Prospective Study
}

\author{
Hyeonjung Yeo, Wonwoo Kim, Hyochun Park, Hoonnam Kim \\ Department of Plastic and Reconstructive Surgery, Daegu Fatima Hospital, Daegu, Korea
}

Background Conscious sedation has been widely utilized in plastic surgery. However, inadequate research has been published evaluating adequate drug dosage and depth of sedation. In clinical practice, sedation is often inadequate or accompanied by complications when sedatives are administered according to body weight alone. The purpose of this study was to identify variables influencing the depth of sedation during conscious sedation for plastic surgery.

Methods This prospective study evaluated 97 patients who underwent plastic surgical procedures under conscious sedation. Serum aspartate aminotransferase (AST), alanine aminotransferase (ALT), creatinine, and glucose levels were measured. Midazolam and ketamine were administered intravenously according to a preset protocol. Bispectral index (BIS) recordings were obtained to evaluate the depth of sedation 4, 10, 15, and 20 minutes after midazolam administration. Associations between variables and the BIS were assessed using multiple regression analysis.

Results Alcohol intake and female sex were positively associated with the mean $\mathrm{BIS}(\mathrm{P}<0.01)$. Age was negatively associated with the mean BIS $(P<0.01)$. Body mass index $(P=0.263)$, creatinine clearance $(P=0.832)$, smoking history $(P=0.398)$, glucose $(P=0.718)$, AST $(P=0.729)$, and ALT $(P=0.423)$ were not associated with the BIS.

Conclusions Older patients tended to have a greater depth of sedation, whereas females and patients with greater alcohol intake had a shallower depth of sedation. Thus, precise dose adjustments of sedatives, accounting for not only weight but also age, sex, and alcohol consumption, are required to achieve safe, effective, and predictable conscious sedation.

Keywords Conscious sedation / Deep sedation / Hypnotics and sedatives / Consciousness monitors
Correspondence: Hyeonjung Yeo Department of Plastic and Reconstructive Surgery, Daegu Fatima Hospital, 99 Ayang-ro, Dong-gu, Daegu 41199, Korea

Tel: +82-53-940-7340

Fax: +82-53-940-7344

E-mail: pleasure-hj@hanmail.net

This article was presented at the Fifth Research \& Reconstructive Forum on May 14, 2015 in Pyeongchang, Korea.

No potential conflict of interest relevant to this article was reported.

Received: 13 Aug $2016 \bullet$ Revised: 20 0ct $2016 \bullet$ Accepted: 20 Oct 2016

pISSN: 2234-6163 • elSSN: 2234-6171 • https://doi.org/10.5999/aps.2017.44.1.5 • Arch Plast Surg 2017;44:5-11

\section{INTRODUCTION}

Conscious sedation has been widely utilized for an increasing number of office-based surgical procedures, and is defined as a drug-induced reduced level of consciousness during which patients respond purposefully to verbal commands, maintain spontaneous respiration, and require no interventions to maintain a patent airway [1]. Conscious sedation has a number of advantages including reduced pain, anxiety, and memories of discomfort related to the surgical procedure. However, sedatives have many possible complications, including hypoxia, hypotension, or hypertension during the procedure, as well as nausea, 
vomiting, or dizziness during the postoperative period. When conscious sedation is performed by surgeons instead of an anesthesiologist, it is critical that the technique be as safe as possible. Many useful articles have been published about the effective and safe management of conscious sedation for plastic surgery [1].

During conscious sedation, the depth of sedation is directly related to the dose of sedative medications. It is important that the minimum dose of sedatives be used to maintain the desired level of sedation. Although various dosing regimens have been suggested, it is not easy for a surgeon to choose the appropriate dose for an individual patient because the sensitivity of patients to sedatives varies considerably. In general, the dose of sedatives is determined based on body weight. However, when sedatives are administered based on body weight alone, sedation is often inadequate or accompanied by complications, such as respiratory depression. In certain patients, administration of a very small dosage of sedative will cause protective airway reflexes to be lost, whereas in others, even high doses cannot achieve adequate sedation.

Many variables can potentially influence a patient's response to sedatives, including age, sex, body mass index (BMI), and liver function [2-4]. The effect of sedatives is generally greater in older patients than in younger patients [3]. Patients with obesity or liver dysfunction have been reported to require adjusted sedative drug doses $[2,4]$. Although several studies have been published about the factors influencing the response to sedative drugs, few prospective studies have examined the relationship between dose and depth of sedation in a clinical setting. The purpose of this study was to identify variables affecting patients' responses to sedative medications during conscious sedation for plastic surgery procedures. This information should help surgeons achieve safe and predictable conscious sedation.

\section{METHODS}

\section{Patient selection}

This study was approved by the Institutional Review Board of Daegu Fatima Hospital (DFH14ORIO248-SR2). It involved patients undergoing conscious sedation for procedures performed by a single plastic surgeon at Daegu Fatima Hospital between October 2014 and October 2015. The inclusion criteria were as follows: (1) American Society of Anesthesiologists physical status I or II, and (2) age of 18-65 years. We excluded patients with contraindications to the sedatives used in this study (midazolam or ketamine), such as an allergic or hypersensitivity reaction to these medications, glaucoma, and previous or current seizures. Ultimately, the study included 97 patients. The cases involved the following types of operations: excisional biopsy (69 cases), facial aesthetic surgery ( 9 cases), local flap surgery ( 8 cases), skin grafting ( 7 cases), and breast and body contouring surgery ( 4 cases).

\section{Perioperative evaluation}

Several variables that can influence the response to sedatives were assessed. Data regarding basic demographics (age and sex), physical characteristics (BMI), lifestyle (alcohol intake, smoking history), comorbidities (e.g., hypertension or diabetes), and medications were collected via a questionnaire. Serum values of aspartate aminotransferase (AST), alanine aminotransferase (ALT), creatinine, and glucose were obtained. Creatinine clearance (Ccr) was calculated.

\section{Perioperative monitoring}

All patients fasted for 4 hours before surgery. Before the administration of any sedatives, monitoring equipment, including a blood pressure cuff, a pulse oximeter, and electrocardiogram leads, were placed on the patient. In addition, three bispectral index (BIS) monitor electrodes were positioned on the patient's forehead, according to the manufacturer's instructions. The BIS is based on the principle that electroencephalography waveforms change with the level of alertness. The BIS objectively quantifies the level of sedation. It provides a single number, which ranges from 0 to 100 . Lower numbers indicate deeper anesthesia (Fig. 1).

The BIS was recorded at baseline (before the administration of any medications) and then at specific intervals during the procedure, as described in the next section. Vital signs, including blood pressure, heart rate, and pulse oxygen saturation

\section{Fig. 1. Level of sedation in relation to the BIS}

90 to 100, awake state; 70 to 90 , light to moderate sedation; 60 to 70 , superficial anesthesia; and 40 to 60 , general anesthesia. BIS, bispectral index.

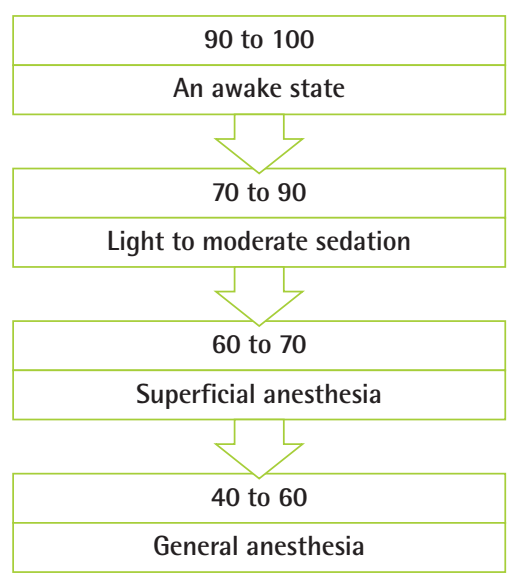


$\left(\mathrm{SpO}_{2}\right)$, were recorded every 10 minutes during surgery. When the $\mathrm{SpO}_{2}$ decreased below $90 \%$, the patient was stimulated verbally. If this did not improve the $\mathrm{SpO}_{2}$, a head tilt, chin lift, or both were performed to maintain a patent airway, and supplemental oxygen was supplied via a nasal cannula, if necessary.

\section{Perioperative sedation protocol and postoperative management}

Sedatives were administered to each patient using a preset protocol. First, $0.04 \mathrm{mg} / \mathrm{kg}$ of intravenous midazolam (Midacum, Myungmoon Pharm., Seoul, Korea) was administered. After 2 minutes, $0.2 \mathrm{mg} / \mathrm{kg}$ of intravenous ketamine (Huons ketamine $\mathrm{HCl}$, Huons Co., Seongnam, Korea) was injected. After waiting an additional 2 minutes to achieve sufficient drug onset, the first recording was obtained. The surgical site was infiltrated with $1 \%$ lidocaine with 1:100,000 epinephrine. Six minutes later, the operation was begun. BIS recordings were then obtained at 10, 15, and 20 minutes after midazolam injection. The Observer's Assessment of Alertness/Sedation (OAA/S) scores (rated from 1 to 5) were determined at the same times. This scoring system was developed to measure the level of alertness in response to verbal and physical stimulation by an observer. The observer speaks the name of the patient in a normal tone and records the score corresponding to the reaction. If the patient does not respond to his or her name spoken in a normal tone, the observer gives a more intense vocal or physical stimulus and records the score corresponding to the reaction (Table 1). After the procedure, the patients were moved to the recovery room. Complications, such as dizziness, nausea, or vomiting, were evaluated using a questionnaire. When the vital signs and level of consciousness were appropriate for discharge, the patients left the recovery room.

\section{Statistical analysis}

Clinical characteristics of the patients were defined as the independent variables (age, sex, BMI, alcohol intake, smoking history, AST, ALT, Ccr, and serum glucose). Mean BIS values at 4, 10,15 , and 20 minutes were defined as the dependent variables. First, simple regression analysis for each variable was used to

\section{Table 1. Observer's Assessment of Alertness and Sedation} (OAA/S) scores

\begin{tabular}{|ll|}
\hline OAA/S & \multicolumn{1}{c|}{ Clinical state } \\
\hline 5 & Responds readily to name spoken in a normal tone \\
4 & Lethargic response to name spoken in a normal tone \\
3 & Responds only after name called loudly and/or repeatedly \\
2 & Does not respond to mild prodding or shaking \\
1 & Does not respond to noxious stimuli \\
\hline
\end{tabular}

predict its effect on the dependent variables. After that, the degree of association between the independent variables and the mean BIS was assessed by multiple regression analysis. Pearson correlation analysis was performed to examine the correlation between the BIS and OAA/S scores at the same measurement time. Additionally, to evaluate how the BIS varied according to $\mathrm{SpO}_{2}$ values, patients were divided depending on whether hypoxia occurred. The mean BIS value was calculated for each group and the independent $t$-test was performed. P-values $<0.05$ were considered to indicate statistical significance. All statistical analyses were performed using SPSS ver. 20.0 (IBM Corp., Armonk, NY, USA).

\section{RESULTS}

A total of 97 patients were evaluated. Each independent variable is expressed as mean \pm standard deviation for continuous variables or as a number (Table 2).

\section{Variables and sedation depth}

The mean BIS of the 97 cases ranged from 59.8 to 97.0, with a mean \pm standard deviation of $83.0 \pm 5$.7. When applying simple regression analysis between the variables and the BIS, age had a statistically significant correlation with the BIS $(\mathrm{P}<0.01)$ (Table 2 ). Using a scatter plot with a regression line, alcohol intake was noted to be positively associated with the BIS (correlation coefficient $=0.127, \mathrm{P}=0.211$ ) (Fig. 2), and age was negatively associated with the BIS (correlation coefficient $=-0.293, \mathrm{P}=0.004$ ) (Fig. 3). For the male sex, the regression coefficient from the simple regression analysis was $-1.999(\mathrm{P}=0.082)$. In other words, both female and patients with greater alcohol intake tended to have a shallower depth of sedation in this study. How-

Table 2. Patient characteristics and simple regression analysis between the variables and the bispectral index

\begin{tabular}{|lccc|}
\hline Variable & Value & R & P-value \\
\hline No. of patients & 97 & - & - \\
Male/Female & $48 / 49$ & 0.177 & 0.082 \\
Age $(\mathrm{yr})$ & $45.5 \pm 11.8$ & 0.293 & $0.004^{\star *}$ \\
Body mass index $\left(\mathrm{kg} / \mathrm{m}^{2}\right)$ & $23.6 \pm 3.1$ & 0.121 & 0.234 \\
Ccr $\left(\mathrm{mL} / \mathrm{min} / 1.73 \mathrm{~m}^{2}\right)$ & $103.3 \pm 31.8$ & 0.025 & 0.802 \\
AST $(\mathrm{mg} / \mathrm{dL})$ & $21.5 \pm 10.7$ & 0.07 & 0.49 \\
ALT $(\mathrm{mg} / \mathrm{dL})$ & $22.5 \pm 19.9$ & 0.091 & 0.371 \\
Glucose $(\mathrm{mg} / \mathrm{dL})$ & $121.1 \pm 100.2$ & 0.013 & 0.896 \\
Alcohol intake (standard drinks/wk) & $5.4 \pm 10.0$ & 0.127 & 0.211 \\
Smoking (pack-year) & $9.6 \pm 18.3$ & 0.056 & 0.524 \\
\hline $\begin{array}{l}\text { Data are mean } \pm \text { standard deviation or number. } \\
\text { Ccr, creatinine clearance; AST, aspartate aminotransferase; }\end{array}$ & ALT, alanine \\
aminotransferase. & & & \\
*P $<0.05,{ }^{* * P}<0.01$. & & \\
\hline
\end{tabular}




\section{Fig. 2. Association between alcohol intake and the BIS}

A positive association was noted between alcohol intake and the BIS (correlation coefficient $=0.127$ ). The relationship was not statistically significant $(P=0.211)$. BIS, bispectral index.

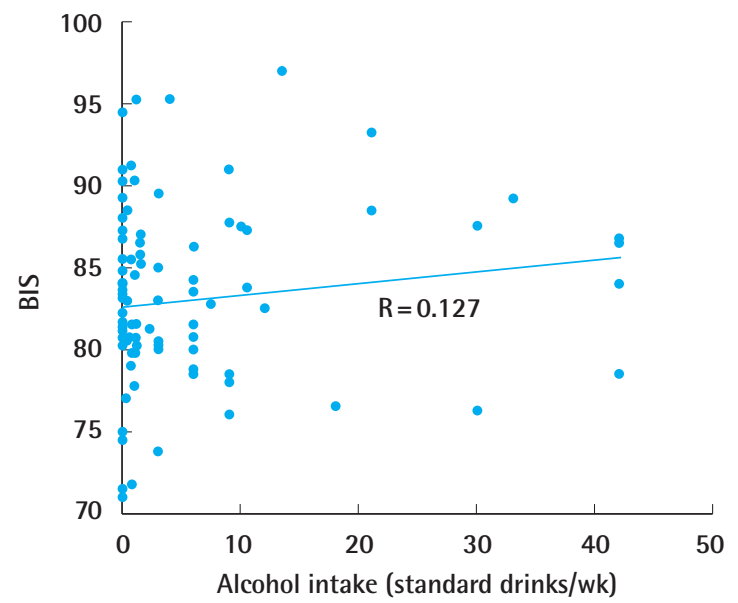

Table 3. Multiple linear regression analysis for the bispectral index

\begin{tabular}{lccccc|}
\hline Variables & Coefficient & SE & $\begin{array}{c}\text { Standardized } \\
\text { Coefficient }\end{array}$ & T & P-value \\
\hline Age & -0.16 & 0.05 & -0.34 & -3 & $0.004^{* *}$ \\
Sex & & & & & \\
$\quad$ Male & -5.07 & 1.41 & -0.45 & -3.6 & $0.001^{* *}$ \\
Female & - & - & - & - & - \\
BMl & 0.25 & 0.23 & 0.14 & 1.13 & 0.26 \\
Ccr & 0.01 & 0.02 & 0.03 & 0.21 & 0.83 \\
Alcohol intake & 0.06 & 0.07 & 0.29 & 2.5 & $0.014^{*}$ \\
Smoking & 0.03 & 0.04 & 0.1 & 0.85 & 0.4 \\
Glucose & 0 & 0.01 & -0.04 & -0.36 & 0.72 \\
AST & 0.02 & 0.06 & 0.04 & 0.35 & 0.73 \\
ALT & 0.03 & 0.04 & 0.11 & 0.81 & 0.42 \\
\hline R & & & & &
\end{tabular}

$R=0.495$ and $R^{2}=0.246$.

SE, standard error; BMI, body mass index; Ccr, creatinine clearance; AST, aspartate aminotransferase; ALT, alanine aminotransferase.

${ }^{*} \mathrm{P}<0.05 ;{ }^{* *} \mathrm{P}<0.01$.

ever, this trend was not statistically significant. However, elderly individuals tended to have a deeper level of sedation, and this trend did reach statistical significance.

The multiple linear regression analysis showed the associations between the mean BIS and all of the variables (Table 3 ). Alcohol intake was positively associated with the BIS (standardized coefficient $=0.29, \mathrm{P}<0.01$ ), age was negatively associated with the BIS (standardized coefficient $=-0.34, \mathrm{P}<0.01$ ), and male sex was negatively associated with the BIS (standardized coefficient $=-0.45, \mathrm{P}<0.01)$. BMI $(\mathrm{P}=0.263), \mathrm{Ccr}(\mathrm{P}=0.832)$, smoking history $(\mathrm{P}=0.398)$, glucose levels $(\mathrm{P}=0.718)$, AST levels $(P=0.729)$, and ALT levels $(P=0.423)$ were not statistically significantly associated with the BIS.

\section{Fig. 3. Association between age and the BIS}

A negative association was noted between age and the BIS (correlation coefficient $=-0.293$ ). The association was statistically significant $(P=0.004)$. BIS, bispectral index.

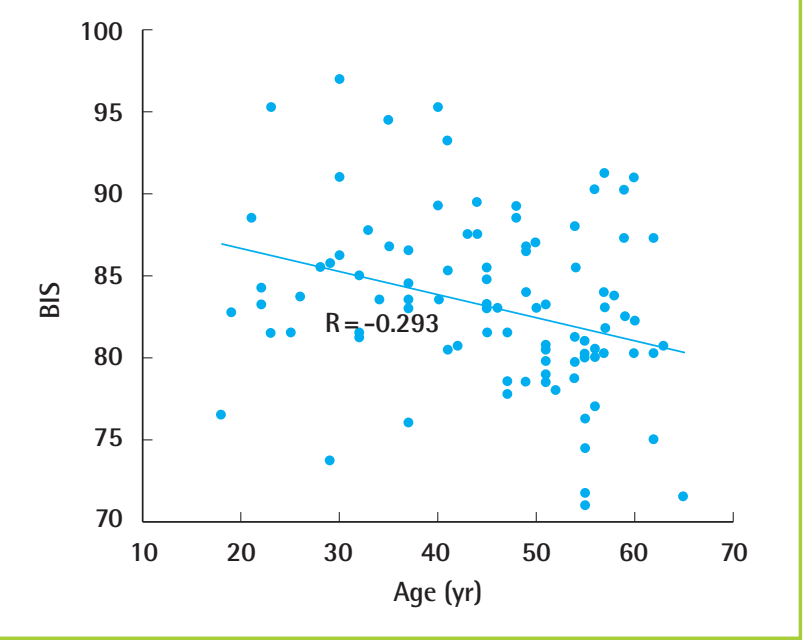

Table 4. BIS in hypoxic and non-hypoxic patients

\begin{tabular}{|c|c|c|c|c|}
\hline \multirow{2}{*}{ Group } & \multirow{2}{*}{ No. $(\%)$} & \multirow{2}{*}{$\frac{\text { BIS }}{\text { Mean } \pm \text { SD }}$} & \multicolumn{2}{|c|}{ T-test } \\
\hline & & & $\mathrm{T}$ & P-value \\
\hline Hypoxic & 12 (12.3) & $81.1 \pm 4.5$ & -1.24 & 0.218 \\
\hline Non-hypoxic & 85 (87.7) & $83.3 \pm 5.8$ & & \\
\hline
\end{tabular}

\section{Complications}

A total of 47 patients (48.4\% of the 97 patients) experienced intraoperative or postoperative adverse events. Postoperative dizziness was the most common complication, occurring in 30 patients (30.9\%). Postoperative nausea and vomiting occurred in 3 patients (3.0\%) and 2 patients (2.0\%), respectively. Intraoperative hypoxia (defined as an $\mathrm{SpO}_{2}$ below 90\%) occurred in 12 patients (12.3\%). Of these, 10 patients $(10.3 \%)$ were treated by a head tilt, chin lift, or both, and 2 patients $(2.0 \%)$ were treated with supplemental oxygen via a nasal cannula. In all patients, the $\mathrm{SpO}_{2}$ rose quickly. The BIS was different in the hypoxic group and the non-hypoxic group (Table 4). The mean BIS for the hypoxic group was $81.1 \pm 4.5$, which was lower than that of the non-hypoxic group $(83.3 \pm 5.8)$; however, this difference was not statistically significant $(\mathrm{P}=0.218)$.

A significant difference was found in the incidence of intraoperative hypoxia depending on whether the Ccr was normal $(\mathrm{P}=0.039)$. Five $(7.5 \%)$ of the 66 patients with a normal $\mathrm{Ccr}$ experienced hypoxia, whereas 7 (22.3\%) of the 31 patients with an abnormal Ccr had hypoxia. Of the latter 7 patients, 3 had chronic kidney disease. In terms of obesity, 2 (6.3\%) of the 32 
Table 5. Correlation coefficients between the BIS and OAA/S scores at the same times

\begin{tabular}{|lc|}
\hline \multirow{2}{*}{ Time $(\min )$} & Correlation coefficient \\
\cline { 2 - 2 } & Between BIS and OAA/S \\
\hline 4 & $0.335^{\star}$ \\
10 & $0.521^{\star}$ \\
15 & $0.507^{\star}$ \\
20 & $0.629^{\star}$ \\
\hline
\end{tabular}

BIS, bispectral index; OAA/S, Observers Assessment of Alertness and Sedation. ${ }^{*} \mathrm{P}<0.001$

overweight patients experienced intraoperative hypoxia, whereas $10(15.3 \%)$ of the 65 non-overweight patients had hypoxia. No significant difference was found in the incidence of hypoxia between the overweight group and the non-overweight group $(\mathrm{P}=0.338)$.

\section{Correlation between the BIS and OAA/S}

The correlation between the BIS and OAA/S at the same measurement time was examined by calculating the correlation coefficient. A positive correlation was found between the BIS and $\mathrm{OAA} / \mathrm{S}$ at all measurement times $(\mathrm{P}<0.001)$ (Table 5). In other words, the depth of sedation as measured by the BIS was consistent with the OAA/S, to a statistically significant extent.

\section{DISCUSSION}

Conscious sedation is widely utilized in plastic surgery, and the demand for it will likely increase in the future because it has many advantages. For safe and effective conscious sedation, several useful guidelines have been published. However, surgeons using sedatives encounter various types of complications in clinical practice, some of which can be serious. In addition, many surgeons find that the response of patients to sedative medications varies, reflecting their differential sensitivity to sedatives. For these reasons, surgeons typically adjust the drug dosage based on their own clinical experience. For example, anecdotal evidence suggests that patients who consume large quantities of alcohol are resistant to sedative medications [5], and a high correlation between patient sensitivity and alcohol intake has been previously reported [6]. Nevertheless, insufficient evidence from high-quality studies has been published regarding which patient populations require adjustments in sedative medications. Thus, we performed the present study to identify variables influencing the depth of sedation achieved during conscious sedation.

Several of the most commonly used agents for conscious sedation are midazolam, ketamine, fentanyl, and propofol. Plastic surgeons commonly use propofol for sedation during surgery. It has a rapid onset and recovery. However, it has several side effects, such as respiratory depression, a hypotensive response, pain with injection, and drug dependence. Midazolam provides anxiolysis and amnesia and has a short duration of action [7]. However, it has no analgesic properties. Ketamine is a dissociative anesthetic, which is relatively safe because it produces minimal cardiorespiratory depression [8]. Ketamine has analgesic effects, which can be synergistically augmented by coadministration with midazolam. Ketamine also significantly reduces the memory of local anesthetic injections and decreases the likelihood of patients being dissatisfied with the operation [9]. For these reasons, we used a combination of midazolam and ketamine in this study. Meanwhile, it is well-known that ketamine causes a paradoxical increase in the BIS [10]. However, reports have suggested that ketamine has no effect on the BIS in low doses [11]. Therefore, it is likely that only midazolam decreased the BIS in this study.

Both the OAA/S and BIS were used in the current study to assess the depth of sedation in the patients. The OAA/S is a commonly used, valid, and reliable index that can directly assess the depth of sedation. However, it has the disadvantage that it involves arousal because the patient is exposed to verbal stimulation, pain stimulation, or both at each measurement time. By contrast, BIS is a relatively new, noninvasive technology that evaluates the level of sedation without the need to arouse the patient. Glass et al. reported that the BIS was an excellent predictor of loss of consciousness in patients receiving midazolam, propofol, or isoflurane [12]. As mentioned above, the BIS can be affected by ketamine. Since no perfect tool has been developed to measure the depth of sedation, both the BIS and OAA/ $S$ were used in this study to obtain relatively reliable results. We showed that the OAA/S scores were correlated with the BIS, which is consistent with the results of a previous study [13]. The author performed a wide variety of surgical procedures. Therefore, the amount of local anesthetic administered differed across operations, which may have affected the BIS. A limitation of this study is that the range of surgery was not controlled.

Sex, age, and alcohol intake were significantly associated with the depth of sedation in the current prospective clinical study. The effect of sex may be related to differences in body composition between men and women. Females have a higher body fat content than males, which leads to a generally higher volume of distribution for lipophilic drugs, such as midazolam, in females. Thus, the same dose per body weight will result in a lower initial plasma concentration of lipophilic drugs in females [14]. It has been previously reported that females recover more quickly from anesthesia and analgesics than males [15], and Yen et al. 
[16] noted that females required more sedatives than males to achieve optimal sedation. Some authors, however, have reported that males may be more likely to exhibit agitation during conscious sedation [1]. In this study, the mean BIS of females was higher than that of males, indicating that the depth of sedation of females tended to be more shallow, in accordance with the results of most previous studies.

Increased age was significantly associated with a deeper level of sedation. In general, the half-life of many drugs, including midazolam, is significantly longer in older patients, and an inverse relationship between age and anesthetic or hypnotic requirements has been reported [17]. Yamashita et al. [18] demonstrated an age-dependent relationship between the BIS and sedation level in patients receiving midazolam sedation. However, Avram et al. [19] demonstrated that changes in midazolam kinetics with age are unlikely to be clinically significant. In this study, the elderly patients generally had a lower BIS, which may be at least partly attributable to reduced drug metabolism with aging.

The association between BIS and alcohol is likely due to crosstolerance between sedative medications and alcohol [5]. Clinically, it is commonly observed that patients who consume large quantities of alcohol are difficult to sedate. Like alcohol, midazolam and ketamine are metabolized in the liver. Thus, it is quite likely that patients who consume considerable quantities of alcohol are difficult to sedate due to the accelerated metabolism of these sedatives. Accordingly, in this study we noted that the more alcohol patients consumed, the higher their BIS was. Therefore, adjustment of the dose of sedatives will be necessary to achieve adequate sedation in individuals with a larger alcohol intake. In addition to alcohol intake, liver function may also affect the response to sedatives. Although it is difficult to directly evaluate liver function, we measured liver enzyme (AST and ALT) levels and observed no significant association between these enzymes and the BIS.

In this study, neither BMI nor Ccr was associated with the depth of sedation. The pharmacokinetics of many drugs are altered in obesity [20]. Renal elimination of drugs is increased in obese patients due to changes in renal blood flow secondary to increased blood volume and cardiac output [4]. The drug distribution might also be different due to marked differences in body composition, and the effects of the drug may thereby be reduced [21]. Patients with a low Ccr will eliminate sedative drugs more slowly, so drug effects would be expected to be more long-lasting, and the dose of these drugs should generally be adjusted [22]. The lack of an association between BMI or Ccr and BIS detected in the current study may reflect our inclusion of subjects who generally had a normal BMI and normal kidney function. Further studies with a larger number of patients with obesity or renal dysfunction will be necessary to more fully evaluate the possibility of such associations.

Other variables not associated with the BIS in the current study included serum glucose and smoking history. Drug metabolism may be altered in diabetic patients or smokers [23,24], which could influence the depth of sedation. However, only 10 diabetic patients were included in our study, and none of those patients had an elevated serum glucose level. For smoking history, we only collected information regarding the pack-years of smoking and not whether the patients were currently smoking. If a substantial number of smokers had stopped smoking, this may have influenced our results.

Regarding complications, patients with reduced kidney function were more likely to exhibit hypoxia. Thus, surgeons should be especially cautious when administering sedatives to patients with decreased kidney function. In contrast, the incidence of respiratory complications was unexpectedly low in obese patients. Several prior studies have reported a relationship between obesity and an increased risk of adverse respiratory events in patients undergoing conscious sedation $[21,25]$. The discrepancy between our results and those of previous studies likely reflects the paucity of obese patients in our study. Most patients with an increased BMI were only mildly to moderately overweight; and few obese patients were included in our study. Regardless of our findings, surgeons should carefully monitor and prepare thoroughly for unexpected adverse events in patients with poor kidney function or obesity.

Dizziness was the most common adverse effect of conscious sedation, and was likely primarily due to midazolam. In all patients, the dizziness improved before leaving the recovery room. In addition, most patients could not recall the pain associated with the local anesthetic injection. This reflects the amnestic qualities of midazolam, and can be considered a positive effect, as it can reduce a patient's fear of undergoing surgery.

As the demand for conscious sedation in plastic surgery increases, sedation-related complications may increase as well. When conscious sedation is performed by surgeons instead of an anesthesiologist, patient safety cannot be overemphasized. Plastic surgeons should fully comprehend conscious sedation and closely monitor all patients undergoing this type of sedation. Especially in critical situations such as respiratory depression, surgeons are responsible for maintaining up-to-date emergency equipment and being well-informed of the protocols necessary to create an environment in which resuscitation can be successfully performed. Additionally, it is the surgeon's duty to obtain a precise and comprehensive patient history and physical examination to achieve sedation safely. More precise protocols 
and/or detailed guidelines regarding the administration of conscious sedation in plastic surgery are clearly necessary.

\section{REFERENCES}

1. Pollock H, Forman S, Pollock T, et al. Conscious sedation/ local anesthesia in the office-based surgical and procedural facility. Clin Plast Surg 2013;40:383-8.

2. Li YH, He R, Ruan JG. Effect of hepatic function on the EC50 of midazolam and the BIS50 at the time of loss of consciousness. J Zhejiang Univ Sci B 2014;15:743-9.

3. Yi Y, Heo D, Son HJ, et al. Association between bispectral index and age and use of sedative drugs in high spinal anaesthesia. J Int Med Res 2013;41:378-85.

4. Brill MJ, Diepstraten J, van Rongen A, et al. Impact of obesity on drug metabolism and elimination in adults and children. Clin Pharmacokinet 2012;51:277-304.

5. Steven RK, Catherine RM, Brian G, et al. Does alcohol consumption impact conscious sedation during endoscopy? Gastrointest Endosc 2001;53:AB84.

6. Mustoe TA, Kim P, Schierle CF. Outpatient abdominoplasty under conscious sedation. Aesthet Surg J 2007;27:442-9.

7. Veselis RA, Reinsel RA, Feshchenko VA. Drug-induced amnesia is a separate phenomenon from sedation: electrophysiologic evidence. Anesthesiology 2001;95:896-907.

8. Rogers R, Wise RG, Painter DJ, et al. An investigation to dissociate the analgesic and anesthetic properties of ketamine using functional magnetic resonance imaging. Anesthesiology 2004;100:292-301.

9. Gruber RP, Morley B. Ketamine-assisted intravenous sedation with midazolam: benefits and potential problems. Plast Reconstr Surg 1999;104:1823-5.

10. Morioka N, Ozaki M, Matsukawa T, et al. Ketamine causes a paradoxical increase in the bispectral index. Anesthesiology 1997;87:A502.

11. Nonaka A, Makino K, Suzuki S, et al. Low doses of ketamine have no effect on bispectral index during stable propofol-remifentanil anesthesia. Masui 2012;61:364-7.

12. Glass PS, Bloom M, Kearse L, et al. Bispectral analysis measures sedation and memory effects of propofol, midazolam, isoflurane, and alfentanil in healthy volunteers. Anesthesiol- ogy 1997;86:836-47.

13. Bell JK, Laasch HU, Wilbraham L, et al. Bispectral index monitoring for conscious sedation in intervention: better, safer, faster. Clin Radiol 2004;59:1106-13.

14. Pleym H, Spigset O, Kharasch ED, et al. Gender differences in drug effects: implications for anesthesiologists. Acta Anaesthesiol Scand 2003;47:241-59.

15. Gan TJ, Glass PS, Sigl J, et al. Women emerge from general anesthesia with propofol/alfentanil/nitrous oxide faster than men. Anesthesiology 1999;90:1283-7.

16. Yen YH, Lin TF, Lin CJ, et al. Sex differences in conscious sedation during upper gastrointestinal panendoscopic examination. J Formos Med Assoc 2011;110:44-9.

17. Katoh T, Suguro Y, Ikeda T, et al. Influence of age on awakening concentrations of sevoflurane and isoflurane. Anesth Analg 1993;76:348-52.

18. Yamashita K, Terao Y, Inadomi C, et al. Age-dependent relationship between bispectral index and sedation level. J Clin Anesth 2008;20:492-5.

19. Avram MJ, Fragen RJ, Caldwell NJ. Midazolam kinetics in women of two age groups. Clin Pharmacol Ther 1983;34: 505-8.

20. Adler M, Schaffner F. Fatty liver hepatitis and cirrhosis in obese patients. Am J Med 1979;67:811-6.

21. Haeck PC, Swanson JA, Iverson RE, et al. Evidence-based patient safety advisory: patient selection and procedures in ambulatory surgery. Plast Reconstr Surg 2009;124:6s-27s.

22. Kidney Disease: Improving Global Outcomes (KDIGO) CKD Work Group. KDIGO 2012 clinical practice guideline for the evaluation and management of chronic kidney disease [Internet]. New York: KDIGO; 2013 [cited 2016 Nov 28]. Available from: http://www.kdigo.org/clinical_practice_guidelines/pdf/CKD/KDIGO_2012_CKD_GL.pdf.

23. Dostalek M, Akhlaghi F, Puzanovova M. Effect of diabetes mellitus on pharmacokinetic and pharmacodynamic properties of drugs. Clin Pharmacokinet 2012;51:481-99.

24. Kroon LA. Drug interactions with smoking. Am J Health Syst Pharm 2007;64:1917-21.

25. Joshi GP, Ahmad S, Riad W, et al. Selection of obese patients undergoing ambulatory surgery: a systematic review of the literature. Anesth Analg 2013;117:1082-91. 